\title{
LOS BELOSTOMATIDOS COMO POTENCIALES CONTROLADORES BIOLOGICOS EN AMBIENTES NO NATURALES
}

Armúa, Aurora Cristina ${ }^{1}$; Ricardo Antonio Rolla ${ }^{2}$

\section{RESUMEN}

Los miembros de la familia Belostomatidae (Insecta: Hemíptera), son predadores incluyendo en su dieta larvas de mosquitos del género Aedes aegypti, Culexquinquefasciatus y caracoles del género Biomphalaria. Estos insectos a pesar de ser eminentemente acuáticos, en las noches de baja presión y altas temperaturas, atraídos por los reflejos de la luz de la ciudad, abandonan los cuerpos de agua, fuera del cual manifiestan gran aptitud para el vuelo, por lo que es frecuente hallarlos en contenedores de agua como las piletas de natación, donde han logrado colonizar y adaptarse a las condiciones físicoquímicas necesarias para el mantenimiento permanente de las mismas. Los objetivos de esta comunicación son, 1-Informar sobre la importancia de los belostomátidos como potenciales controladores de larvas de interés sanitario. 2-Evaluar la tolerancia de los belostomatidos frente a condiciones físico-químicas diferentes al ambiente natural. El trabajo se inició haciendo relevamientos de domicilios, ubicados en el área urbana de la ciudad de Corrientes que contaran con piletas de natación de mantenimiento permanente y la presencia de belostomátidos en las mismas. El hallazgo de larvas o pupas por piletas se consideró como foco de presencia del mosquito adulto. Se seleccionaron cuatro piletas de natación, para el estudio. Las variables físicoquímicas registradas en cada uno de los controles fueron temperatura, $\mathrm{pH}$ y cloro residual. Los datos fueron registrados en planillas. Los resultados hasta el momento demuestran que Belostoma elegans y Belostoma lopretoae fueron las especies más frecuentes halladas en las piletas de natación.

Palabras claves: Control biológico-Belostoma-predación-importancia sanitaria -dípteros

\section{INTRODUCCION}

El control biológico es una herramienta importante dentro del manejo integrado de plagas, dado las ventajas que ofrece sobre otros métodos como el control químico, al minimizar los riesgos ambientales asociados a plaguicidas. Los belostomátidos, conocidos vulgarmente como "Chinches de agua" son muy frecuentes en ambientes acuáticos de la provincia de Corrientes. En noches de baja presión y altas temperatu-

\footnotetext{
${ }^{1}$ Dra. En Biología. Facultad de Ciencias Exactas y Naturales y Agrimensura (UNNE)Av.Libertad 5470 - 3400 - Corrientes - email: acarmua@exa.unne.edu.ar

${ }^{2}$ Lic. En Química. Ministerio de Salud Pública - Dirección de Saneamiento AmbientalCórdoba 609 - 3400 - Corrientes - email:lilorolla@hotmail.com
} 
ras suelen abandonar los cuerpos de agua naturales atraídos por los reflejos de la luz de la zona céntrica de la ciudad. Debido a esto, es frecuente hallarlos en el interior de recipientes con agua y piletas de natación de mantenimiento permanente ubicado en patios de viviendas, donde han logrado colonizar y adaptarse a las condiciones físico-químico de las mismas.Estos insectos se caracterizan por ser predadores eficientes tanto de invertebrados como de vertebrados acuáticos, (Menke, 1979), incluyendo en su dieta larvas de Dípteros (mosquitos) importantes como vectores de enfermedades, Paludismo, Fiebre Amarilla, Dengue entre otras. Se alimentan también de caracoles, (SMITH, 1997) del género Biomphalaria como B. tenagophila y B. straminea, (Armúa, y Estévez, 2005), importantes por ser hospedadores intermediario del parásito causante de la esquistosomiasis enfermedad que afecta al hombre y otros vertebrados,por lo cual es este un aspecto interesante que presenta el estudio de este grupo, ya que constituyen una alternativa a tener presente en la lucha del control biológico. Los antecedentes sobre este tema son muy escasos, entre los autores que han efectuado aportes sobre el comportamiento alimentario de los belostomátidos se citan a, Consolli et al.(1989), determinaron la alimentación de Belostoma micantulum sobre larvas y pupas de Aedes fluviatilis. Armúa y Estévez (1998) (a) estudiaron el comportamiento alimentario en los estadios preimaginales de B. elegans, en condiciones controladas, Kehr y Schnack (1991), realizaron un trabajo referido a las variaciones en las tasas de predación, en función del tamaño de las presas (larvas de anuro Bufo arenarum) expuestas a la presencia de larvas del IV y V estadio y adultos de Belostoma oxyurum.

\section{OBJETIVOS}

1- Informar sobre la importancia de los belostomátidos como potenciales controladores de larvas de interés sanitario.

2- Evaluar la tolerancia de los belostomatidos frente a condiciones físicoquímicas diferente al ambiente natural.

\section{METODOLOGIA}

El trabajo se inició haciendo un relevamiento de domicilios, ubicados en el área urbana de la ciudad de corrientes que contaran con piletas de natación de mantenimiento permanente y la presencia de belostomátidos en las mismas. El hallazgo de larvas o pupas de mosquitos por piletas se consideró como foco de presencia del mosquito adulto.Se seleccionaron cuatro piletas de natación en viviendas donde fueron halladas larvas de Aedes aegypti. (determinación sistemática a cargo de especialistas en el tema Dengue).

Se solicitóó autorización de los propietarios para realizar las observaciones del comportamiento alimentario de los belostomátidos. Los registros realizados durante las visitas semanales a los domicilios seleccionados consistieron en el control de los parámetros físicos químicos y bacteriológicos, siendo los mismos $\mathrm{pH}$, cloro residual total (por método calorimétrico de ortotolidina) y temperatura del ambiente y del agua del natatorio con termómetro digital.Los datos fueron registrados en planillas elaboradas para tal fin. Se determinó presencia-ausencia de belostomatidos y larvas de dípteros de importancia sanitaria. 


\section{RESULTADOS}

$\mathrm{E}$ l trabajo de investigación que se viene realizando desde 2008 en la Facultad de Ciencias Exactas y Naturales y Agrimensura de la Universidad Nacional del Nordeste con el grupo de insectos perteneciente a la familia Belostomatidae, nos ha permitido obtener resultados significativos en condiciones de laboratorio, en lo referente al control de larvas de dípteros. Si bien los belostomátidos, como otros insectos predadores, se alimentan indistintamente de larvas de insectos dañinos como de larvas de insectos benéficos, las especies Belostoma loprettoae Estévez y Armúa sp.nov.; y Belostoma elegans Mayr; han demostrado preferencia alimentaria superior al $87 \%$ por

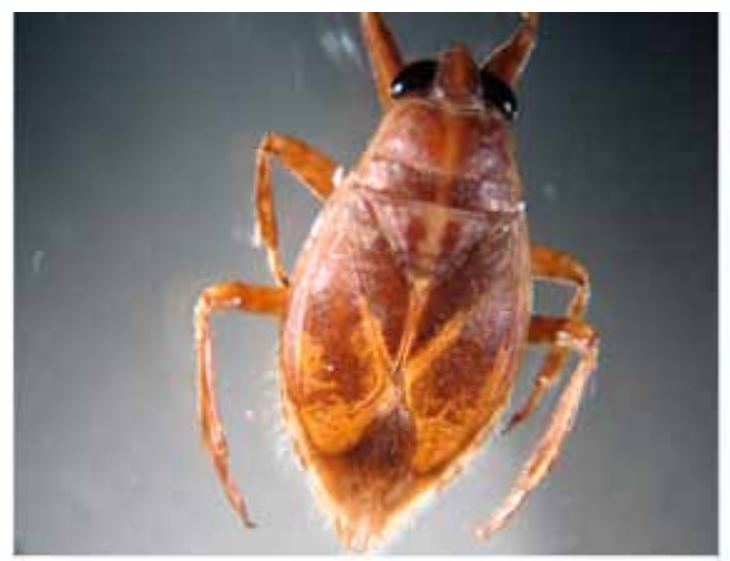

Fig I Belostoma lopretoae: I ista dorsal

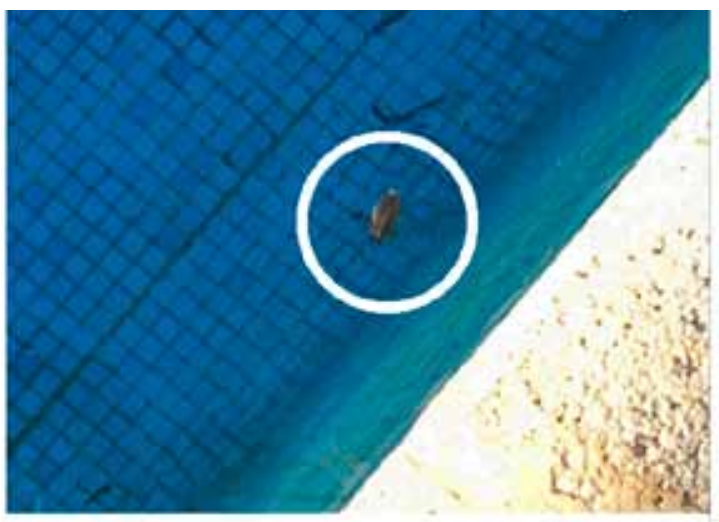

Fig 3B.lopretoae ubicado en la pared del natatorio larvas de Aedes aegyptis, Culexquinquefasciatus, frente a otras presas que habitualmente ocupan el mismo ambiente, independiente de la densidad de las presas suministradas como alimento. En las piletas la presencia de larvas de Aedes aegyptis, fue disminuyendo con el aumento de los ejemplares de belostomatidos lo que permitió dejar de usar BTI (Bacilusturigiensisisraeliensis) en las piletas donde se realizaron los estudios.

Belostoma loprettoae (fig. 1, 2 y 3) ha tolerado perfectamente concentraciones de cloro residual superior a $2,00 \mathrm{mg} / \mathrm{li}$ tro. Rango de $\mathrm{pH}$ entre 7,3 a 8,0. El número de belostomatidos por natatorio osciló entre un mínimo de 10 y un máximo de 15, concentrándose mayormente en

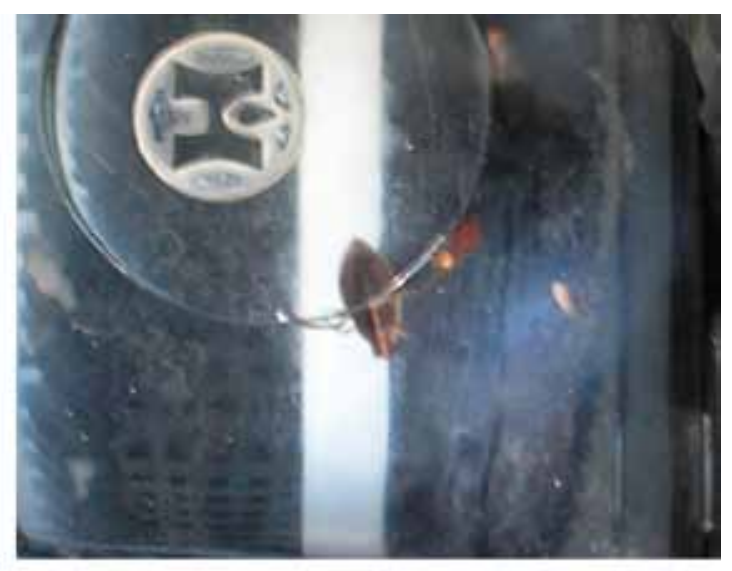

Fig 2B.lopretoae en filtro de pelo de la bomba

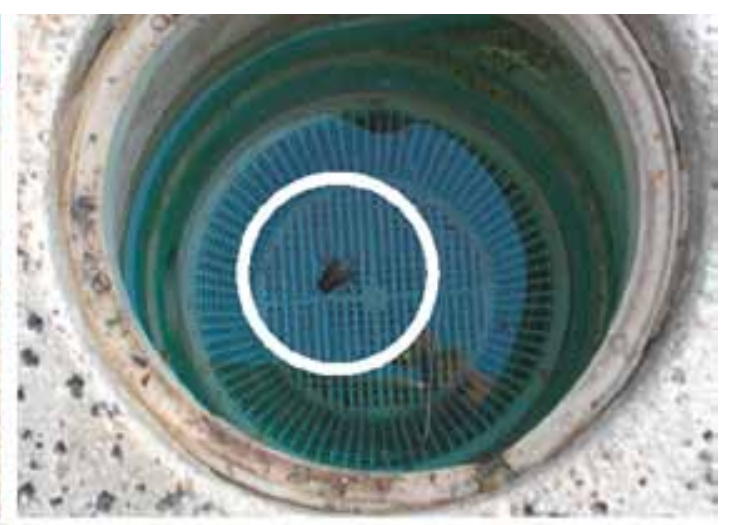

Fig 4 Belostoma hallado en zona del skimmer (A) 
la zona del skimmer (pre filtro superficial donde se lleva a cabo la eliminación de todo el material en suspensión). (fig.4)

Este comportamiento nos permite su- gerir a la población en general, la conservación de estos insectos predatores, que no ocasionan daño alguno, y colaboran a disminuir los estadios inmaduros de dípteros de importancia sanitaria en natatorios.

\section{BIBLIOGRAFIA}

- Armúa, A.C. y A.L. Estévez. 1998. Relevamiento de la fauna de Belostomatidae en la provincia de Corrientes. FACENA. Vol. 14. Pág. $71-74$

- Armúa, A. C. y A. L. Estévez. 2005. "Predation on Biomphalaria sp. (Mollusca: Planorbidae) by three species of the genus Belostoma (Hemiptera: Belostomatidae)". Brazilian Journal of Biology. 66(4): 1033-1035 ISSN 034 7108 .

- Consolli, R.A.; M.H. Pereira, A. Lane de Melo y L.H.Pereira 1989. BelostomamicantulumStal,1858. (Hemiptera:Belostomatidae) as a predator of larvae and pipae of Aedes-
Aluviatilis (Diptera:Culicidae) in laboratory conditions. Mem.Inst.Oswaldo Cruz 84(4):577-578.

- Kehr,A.I. y J.A. Schnack (1991).Predatoryprey relationship between wáter bugs (Belostoma oxyurum) and larval anurans (Bufoarenarum). Alytes 9 (3):61-69.

- Menke, A.S. (1979). Family Belostomatidae-giant wáter bugs, electric lightbugs:toebiters,pp76-86.In A.S. Menke (ed) The semiaquatic and aquatic Hemiptera of California (Heteroptera:Hemiptera). Bull. Calif. Insect Survey. 21.Univ. calif.Press.Berkeley. 